Proyecciones Journal of Mathematics

Vol. 35, No 3, pp. 235-244, September 2016.

Universidad Católica del Norte

Antofagasta - Chile

\title{
An alternative proof of a Tauberian theorem for Abel summability method
}

\author{
İbrahim Çanak \\ Ege University, Turkey \\ and \\ Ümit Totur \\ Adnan Menderes University, Turkey \\ Received: December 2014. Accepted : July 2016
}

\begin{abstract}
Using a corollary to Karamata's main theorem [Math. Z. 32 (1930), 319-320], we prove that if a slowly decreasing sequence of real numbers is Abel summable, then it is convergent in the ordinary sense.
\end{abstract}

Subjclass [2010] : 40A05; 40E05; 40G10.

Keywords : Abel summability, slowly decreasing sequences, Tauberian conditions and theorems. 


\section{Introduction}

A number of authors such as Schmidt [9], Maddox [6], Móricz [8], and Talo and Başar [11] have proved several Tauberian theorems for some summability methods for which slowly decreasing condition for sequences is a Tauberian condition. Schmidt [9] obtained that the slowly decreasing condition for sequences of real numbers is a Tauberian condition for Abel summability. Maddox [6] introduced the slowly decreasing sequence in an ordered linear space and proved that a Cesàro summable sequence is convergent if it is slowly decreasing in an ordered linear space. Móricz [8] established a Tauberian theorem which states that ordinary convergence of a sequence follows from its statistical Cesàro summability if it is slowly decreasing. Talo and Başar [11] introduced the concept of slowly decreasing sequences for fuzzy numbers and they proved that the slowly decreasing condition for sequences is a Tauberian condition for the statistical convergence and Cesàro summability for sequences of fuzzy numbers.

Littlewood [5] proved that $n\left(u_{n}-u_{n-1}\right)=O(1)$ is a Tauberian condition for Abel summability of $\left(u_{n}\right)$. But his proof was complicated and based on the repeated differentiation. A first clever and surprisingly simple proof based on Weierstrass approximation theorem of Littlewood's theorem was given by Karamata [2].

The main purpose of this study is to give an alternative simpler proof of the following Tauberian theorem which is more general than Littlewood's theorem [5] for Abel summability method.

Theorem 1.1. If $\left(u_{n}\right)$ is Abel summable to $s$ and slowly decreasing, then $\lim _{n} u_{n}=s$.

To prove Theorem 1.1, we first obtain Cesàro convergence of the generator sequence of a given sequence $\left(u_{n}\right)$ by means of a corollary to Karamata's main Theorem, and then recover convergence of $\left(u_{n}\right)$ by Tauber's second theorem [12].

Our proof is much easier than the existing one and uses the well known results in Tauberian theory. For a different proof of Theorem 1.1, see [1]. 


\section{Preliminaries}

For a sequence $u=\left(u_{n}\right)$ of real numbers, we write $\left(u_{n}\right)$ in terms of $\left(v_{n}\right)$ as

$$
u_{n}=v_{n}+\sum_{k=1}^{n} \frac{v_{k}}{k}+u_{0}, \quad(n=1,2, \ldots)
$$

where $v_{n}=\frac{1}{n+1} \sum_{k=1}^{n} k\left(u_{k}-u_{k-1}\right)$. The sequence $\left(v_{n}\right)$ is called a generator sequence of $\left(u_{n}\right)$. We note that $\sigma_{n}^{(1)}(u)=\frac{1}{n+1} \sum_{k=0}^{n} u_{k}=u_{0}+\sum_{k=1}^{n} \frac{v_{k}}{k}$.

Let $u=\left(u_{n}\right)$ be a sequence of real numbers. For each nonnegative integer $m$, we define $\sigma_{n}^{(m)}(u)$ by

$$
\sigma_{n}^{(m)}(u)= \begin{cases}\frac{1}{n+1} \sum_{k=0}^{n} \sigma_{k}^{(m-1)}(u) & , m \geq 1 \\ u_{n} & , m=0\end{cases}
$$

A sequence $\left(u_{n}\right)$ is said to be Abel summable to $s$ if $u_{0}+\sum_{n=1}^{\infty}\left(u_{n}-\right.$ $\left.u_{n-1}\right) x^{n}$ converges for $0<x<1$, and tends to $s$ as $x \rightarrow 1^{-}$.

A sequence $\left(u_{n}\right)$ is called $(A, m)$ summable to $s$ if $\left(\sigma_{n}^{(m)}(u)\right)$ is Abel summable to $s$. If $m=0$, then $(A, m)$ summability reduces to Abel summability. It is clear that Abel summability of $\left(u_{n}\right)$ implies $(A, m)$ summability of $\left(u_{n}\right)$.

Throughout this work, the symbol $[\lambda n]$ denotes the integral part of the product $\lambda n$.

A sequence $\left(u_{n}\right)$ is said to be slowly decreasing [9] if

$$
\lim _{\lambda \rightarrow 1^{+}} \liminf _{n \rightarrow \infty} \min _{n+1 \leq k \leq[\lambda n]}\left(u_{k}-u_{n}\right) \geq 0
$$

or equivalently [8],

$$
\lim _{\lambda \rightarrow 1^{-}} \liminf _{n \rightarrow \infty} \min _{[\lambda n]+1 \leq k \leq n}\left(u_{n}-u_{k}\right) \geq 0 .
$$

Notice that $\left(u_{n}\right)$ is slowly decreasing if the classical one-sided Tauberian condition of Landau [1] is satisfied, that is, there exists a positive constant $C>0$ such that

$$
n\left(u_{n}-u_{n-1}\right) \geq-C
$$

for all nonnegative $n$. Indeed, for any $k>n$, we have

$$
u_{k}-u_{n}=\sum_{j=n+1}^{k}\left(u_{j}-u_{j-1}\right) \geq-C \sum_{j=n+1}^{k} \frac{1}{j} \geq-C \log \left(\frac{k}{n}\right)
$$


whence we conclude that

$$
\liminf _{n \rightarrow \infty} \min _{n+1 \leq k \leq[\lambda n]}\left(u_{k}-u_{n}\right) \geq-C \log \lambda, \quad \lambda>1 .
$$

Taking $\lambda \rightarrow 1^{+}$, we have the inequality (2.2).

Note that we used $C$ to denote a constant, possibly different at each occurrence.

A sequence $\left(u_{n}\right)$ is slowly increasing if and only if $\left(-u_{n}\right)$ is slowly decreasing, and an equivalent definition of a slowly increasing sequence as follows:

A sequence $\left(u_{n}\right)$ is said to be slowly increasing if

$$
\lim _{\lambda \rightarrow 1^{+}} \limsup _{n \rightarrow \infty} \max _{n+1 \leq k \leq[\lambda n]}\left(u_{k}-u_{n}\right) \leq 0 .
$$

The condition (2.5) is reformulated as follows (see [8]):

$$
\lim _{\lambda \rightarrow 1^{-}} \limsup _{n \rightarrow \infty} \max _{[\lambda n]+1 \leq k \leq n}\left(u_{n}-u_{k}\right) \leq 0 .
$$

It is plain that a sequence $\left(u_{n}\right)$ is said to be slowly oscillating if and only if $\left(u_{n}\right)$ is both slowly increasing and slowly decreasing. Notice that each of the conditions (2.2) and (2.5) is necessary for convergence (see [4]).

If $\left(u_{n}\right)$ converges to $s$, then $\left(u_{n}\right)$ is Abel summable to $s$. However, the converse of this statement is not always true. Note that Abel summability of $\left(u_{n}\right)$ implies convergence of $\left(u_{n}\right)$ under certain additional hypotheses called Tauberian conditions. Any theorem which states that convergence of sequence $\left(u_{n}\right)$ follows from Abel summability of $\left(u_{n}\right)$ and some Tauberian condition(s) is called a Tauberian theorem for Abel summability method.

\section{Corollary to Karamata's Main Theorem and Lemmas}

Our proof is based on the following corollary to Karamata's main theorem and three Lemmas.

Corollary to Karamata's Main Theorem. ([2]) If $u=\left(u_{n}\right)$ is Abel summable to $s$ and $u_{n} \geq-C$ for some nonnegative $C$, then $\lim _{n} \sigma_{n}^{(1)}(u)=s$.

Lemma 3.1. ([3]) If, for $x \rightarrow 1^{-}$, a function $f(x)$, which is integrable in $[0,1]$, satisfies the limiting relation

$$
(1-x)^{2} f(x) \rightarrow s
$$


then, for $x \rightarrow 1^{-}$, we also have

$$
(1-x) \int_{0}^{x} f(t) d t \rightarrow s .
$$

The next lemma gives a necessary condition for a slowly decreasing sequence in terms of the generator sequence $\left(v_{n}\right)$.

Lemma 3.2. ([7]) If $\left(u_{n}\right)$ is slowly decreasing, then $v_{n} \geq-C$ for some $C$, where $v_{n}=\frac{1}{n+1} \sum_{k=1}^{n} k\left(u_{k}-u_{k-1}\right)$.

Next, we represent the difference $u_{n}-\sigma_{n}^{(1)}(u)$ in two different ways.

Lemma 3.3. ([10]) Let $u=\left(u_{n}\right)$ be a sequence of real numbers.

(i) For $\lambda>1$ and sufficiently large $n$,

$$
u_{n}-\sigma_{n}^{(1)}(u)=\frac{[\lambda n]+1}{[\lambda n]-n}\left(\sigma_{[\lambda n]}^{(1)}(u)-\sigma_{n}^{(1)}(u)\right)-\frac{1}{[\lambda n]-n} \sum_{k=n+1}^{[\lambda n]}\left(u_{k}-u_{n}\right) .
$$

(ii) For $0<\lambda<1$ and sufficiently large $n$,

$u_{n}-\sigma_{n}^{(1)}(u)=\frac{[\lambda n]+1}{n-[\lambda n]}\left(\sigma_{n}^{(1)}(u)-\sigma_{[\lambda n]}^{(1)}(u)\right)+\frac{1}{n-[\lambda n]} \sum_{k=[\lambda n]+1}^{n}\left(u_{n}-u_{k}\right)$.

\section{Proof of Theorem 1.1}

Proof. Since $\left(u_{n}\right)$ is Abel summable to $s$, then $\left(\sigma_{n}^{(1)}(u)\right)$ is also Abel summable to $s$. Hence, we conclude by $(2.1)$ that $\left(v_{n}\right)=\left(\frac{1}{n+1} \sum_{k=0}^{n} k\left(u_{k}-u_{k-1}\right)\right)$ is Abel summable to zero by Lemma 3.1. It follows by Lemma 3.2 that there exists a nonnegative $C$ such that

$$
v_{n} \geq-C
$$

Taking (4.1) and the fact that $\left(v_{n}\right)$ is Abel summable to zero into account, we obtain by Corollary to Karamata's Main Theorem that $\sigma_{n}^{(1)}(v)=$ 
$o(1)$ as $n \rightarrow \infty$. Since $\left(\sigma_{n}^{(1)}(u)\right)$ is Abel summable to $s$ and $\sigma_{n}^{(1)}(v)=o(1)$ as $n \rightarrow \infty$, we have that $\left(\sigma_{n}^{(1)}(u)\right)$ converges to $s$ by Tauber's second theorem $[12]$.

By the fact that every convergent sequence is slowly increasing, we have

$\left(\sigma_{n}^{(1)}(u)\right)$ is slowly increasing. Thus, $\left(-\sigma_{n}^{(1)}(u)\right)$ is slowly decreasing. Since $\left(s_{n}\right)$ is slowly decreasing, $\left(v_{n}\right)$ is slowly decreasing.

By Lemma 3.3 (i), we have

$$
v_{n}-\sigma_{n}^{(1)}(v)=\frac{[\lambda n]+1}{[\lambda n]-n}\left(\sigma_{[\lambda n]}^{(1)}(v)-\sigma_{n}^{(1)}(v)\right)-\frac{1}{[\lambda n]-n} \sum_{k=n+1}^{[\lambda n]}\left(v_{k}-v_{n}\right) .
$$

It is easy to verify that for $\lambda>1$ and sufficiently large $n$,

$$
\frac{\lambda}{2(\lambda-1)} \leq \frac{[\lambda n]+1}{[\lambda n]-n} \leq \frac{3 \lambda}{2(\lambda-1)} .
$$

By $\sigma_{n}^{(1)}(v)=o(1)$ as $n \rightarrow \infty$ and (4.10), for all $\lambda>1$,

$$
\lim _{n \rightarrow \infty} \frac{[\lambda n]+1}{[\lambda n]-n}\left(\sigma_{[\lambda n]}^{(1)}(v)-\sigma_{n}^{(1)}(v)\right)=0 .
$$

By (4.2) and (4.3), we have

$$
v_{n}-\sigma_{n}^{(1)}(v) \leq \frac{[\lambda n]+1}{[\lambda n]-n}\left(\sigma_{[\lambda n]}^{(1)}(v)-\sigma_{n}^{(1)}(v)\right)-\min _{n+1 \leq k \leq[\lambda n]}\left(v_{k}-v_{n}\right) .
$$

Taking limsup of both sides of (4.5), we have

$$
\begin{aligned}
\limsup _{n}\left(v_{n}-\sigma_{n}^{(1)}(v)\right) & \leq \limsup _{n \rightarrow \infty} \frac{[\lambda n]+1}{[\lambda n]-n}\left(\sigma_{[\lambda n]}^{(1)}(v)-\sigma_{n}^{(1)}(v)\right) \\
& -\liminf _{n} \min _{n+1 \leq k \leq[\lambda n]}\left(v_{k}-v_{n}\right) .
\end{aligned}
$$


The inequality (4.6) becomes

$$
\limsup _{n}\left(v_{n}-\sigma_{n}^{(1)}(v)\right) \leq-\liminf _{n} \min _{n+1 \leq k \leq[\lambda n]}\left(v_{k}-v_{n}\right)
$$

by (4.4). Taking $\lambda \rightarrow 1^{+}$in (4.7), we have

$$
\limsup _{n}\left(v_{n}-\sigma_{n}^{(1)}(v)\right) \leq 0
$$

by $(2.2)$.

By Lemma 3.3 (ii), we have

$$
v_{n}-\sigma_{n}^{(1)}(v)=\frac{[\lambda n]+1}{n-[\lambda n]}\left(\sigma_{n}^{(1)}(v)-\sigma_{[\lambda n]}^{(1)}(v)\right)+\frac{1}{n-[\lambda n]} \sum_{k=[\lambda n]+1}^{n}\left(v_{n}-v_{k}\right) .
$$

It is easy to verify that for $0<\lambda<1$ and sufficiently large $n$,

$$
\frac{\lambda}{2(1-\lambda)} \leq \frac{[\lambda n]+1}{n-[\lambda n]} \leq \frac{3 \lambda}{2(1-\lambda)}
$$

By $\sigma_{n}^{(1)}(v)=o(1)$ as $n \rightarrow \infty$ and (4.10), for all $0<\lambda<1$,

$$
\lim _{n \rightarrow \infty} \frac{[\lambda n]+1}{n-[\lambda n]}\left(\sigma_{n}^{(1)}(v)-\sigma_{[\lambda n]}^{(1)}(v)\right)=0 .
$$

By (4.9) and (4.10), we have

$$
v_{n}-\sigma_{n}^{(1)}(v) \geq \frac{[\lambda n]+1}{n-[\lambda n]}\left(\sigma_{n}^{(1)}(v)-\sigma_{[\lambda n]}^{(1)}(v)\right)+\min _{[\lambda n]+1 \leq k \leq n}\left(v_{n}-v_{k}\right) .
$$

Taking lim inf of both sides of (4.12), we have

$$
\begin{aligned}
\liminf _{n}\left(v_{n}-\sigma_{n}^{(1)}(v)\right) & \geq \liminf _{n} \frac{[\lambda n]+1}{n-[\lambda n]}\left(\sigma_{n}^{(1)}(v)-\sigma_{[\lambda n]}^{(1)}(v)\right) \\
& +\liminf _{n} \min _{[\lambda n]+1 \leq k \leq n}\left(v_{n}-v_{k}\right) .
\end{aligned}
$$


The inequality (4.13) becomes

$$
\liminf _{n}\left(v_{n}-\sigma_{n}^{(1)}(v)\right) \geq \liminf _{n} \min _{[\lambda n]+1 \leq k \leq n}\left(v_{n}-v_{k}\right)
$$

by (4.11).

Taking $\lambda \rightarrow 1^{-}$in (4.14), we have

$$
\liminf _{n}\left(v_{n}-\sigma_{n}^{(1)}(v)\right) \geq 0
$$

by $(2.3)$.

Combining (4.8) and (4.15) yields that $v_{n}=o(1)$ as $n \rightarrow \infty$. Since $\left(u_{n}\right)$ is Abel summable to $s$ and $v_{n}=o(1)$ as $n \rightarrow \infty, \lim _{n} u_{n}=s$ by Tauber's second theorem [12]. This completes the proof.

Using Theorem 1.1, we show that slow decrease of $\left(u_{n}\right)$ is also a Tauberian condition for $(A, m)$ summability method.

Theorem 4.1. If $\left(u_{n}\right)$ is $(A, m)$ summable to $s$ and slowly decreasing, then $\lim _{n} u_{n}=s$.

Proof. Let $\left(u_{n}\right)$ be slowly decreasing. Then, we have $v_{n} \geq-C$ for some $C$ by Lemma 3.2. Since $n\left(\sigma_{n}^{(1)}(u)-\sigma_{n-1}^{(1)}\right)=v_{n}$ for all nonnegative $n$, we conclude that $\left(\sigma_{n}^{(1)}(u)\right)$ is slowly decreasing if we replace $u_{n}$ in (2.4) by $\sigma_{n}^{(1)}(u)$.

It easily follows that $\left(\sigma_{n}^{(m)}(u)\right)$ is slowly decreasing for each nonnegative $m$.

Since $\left(u_{n}\right)$ is $(A, m)$ summable to $s$, we have

$$
\lim _{n} \sigma_{n}^{(m)}(u)=s
$$

by Theorem 1.1. By definition, we have

$$
\sigma_{n}^{(m)}(u)=\sigma_{n}^{(1)}\left(\sigma^{(m-1)}(u)\right)
$$

From (4.16) and (4.17) it follows that $\left(u_{n}\right)$ is $(A, m-1)$ summable to $s$. Since $\left(\sigma_{n}^{(m-1)}(u)\right)$ is slowly decreasing, we have $\lim _{n} \sigma_{n}^{(m-1)}(u)=s$ by Theorem 1.1. Continuing in this way, we obtain that $\lim _{n} u_{n}=s$. 


\section{References}

[1] G. H. Hardy, Divergent series, Oxford University Press, (1948).

[2] J. Karamata, Über die Hardy-Littlewoodschen Umkehrungen des Abelschen Stetigkeitssatzes, Math. Z., 32, pp. 319-320, (1930).

[3] K. Knopp, Theory and application of infinite series, Dover Publications, (1990).

[4] J. Korevaar, Tauberian theory, Springer, 2004.

[5] J. E. Littlewood, The converse of Abel's theorem on power series, London M. S. Proc. 2 (9), pp. 434-448, (1911).

[6] I. J. Maddox, A Tauberian theorem for ordered spaces, Analysis, 9, (3), pp. 297-302, (1989).

[7] G. A. Mikhalin, Theorem of Tauberian type for $\left(J, p_{n}\right)$ summation methods, Ukrain. Mat. Zh. 29 (1977), 763-770. English translation: Ukrain. Math. J. 29, pp. 564-569, (1977).

[8] F. Móricz, Ordinary convergence follows from statistical summability $(C, 1)$ in the case of slowly decreasing or oscillating sequences, Colloq. Math. 99, (2), pp. 207-219, (2004).

[9] R. Schmidt, Über divergente Folgen und lineare Mittelbildungen, Math. Z. 22 (1), pp. 89-152, (1925).

[10] Č. V. Stanojević, V. B. Stanojević, Tauberian retrieval theory, Publ. Inst. Math. (Beograd) (N.S.) 71 (85), pp. 105-111, (2002).

[11] Ö. Talo, F. Başar, On the slowly decreasing sequences of fuzzy numbers, Abstr. Appl. Anal. Art. ID 891986, 7, pp. ..., (2013).

[12] A. Tauber, Ein satz aus der theorie der unendlichen reihen, Monatsh. f. Math. u. Phys. 7, pp. 273-277, (1897). 
İbrahim Çanak

Department of Mathematics

Ege University

Izmir

Turkey

e-mail : ibrahim.canak@ege.edu.tr

and

Ümit Totur

Department of Mathematics

Adnan Menderes University

Aydin

Turkey

e-mail : utotur@adu.edu.tr 